NEUER BRUCKENBERGER-BERICHT 2009

\title{
Herznotfallambulanzen am falschen Ort?
}

— Inzwischen gibt es in Deutschland 92 zertifizierte Chest-Pain-Units (CPUs). Doch ein Blick auf die neuen Bruckenberger-Zahlen zeigt: Dort wo die Sterblichkeit am höchsten ist, fehlen derzeit noch solche Ambulanzen zur Erkennung eines Herznotfalls.

„Gerade in Gebieten, in denen sehr viele Menschen an Herzkrankheiten sterben, wurden leider nur wenige CPUs eingerichtet. Dies betrifft Niedersachen, Thüringen, Sachsen-Anhalt, Sachsen, Brandenburg und Mecklenburg-Vorpommern“, erläuterte Prof. Thomas Meinertz, Ham- burg. „Andererseits wurden in Regionen CPUs eingerichtet, deren Herzinfarktversorgung ohnehin schon gut ist", so Meinertz. Das Defizit in den unterversorgten Regionen müsse korrigiert werden. Daten aus Deutschland, den USA und Großbritannien belegen nämlich, dass die Einrichtung von CPUs Sterblichkeit und Liegedauer von Infarktpatienten deutlich verringern. Auch schwerwiegende Komplikationen wie Herzinsuffizienz lassen sich durch CPUs vermeiden.

RK -

Quelle: Pressemitteilung 29/2010 der Deutschen Herzstiftung vom 1. Oktober 2010

\section{INTERAKTIVE VIDEOSPIELE}

\section{Hohe Verletzungsgefahr}

— Interaktive Videospiele verlangen vollen Körpereinsatz. Dies ist gerade bei Kindern mit einer nicht unerheblichen Verletzungsgefahr verbunden, warnen Pädiater. Innerhalb von fünf Jahren registrierte das National Electronic Injury Surveillance System 696 Verletzungen beim Videospielen.

604 Verletzungen hatten sich Spieler mit den traditionellen Videospielen zugezogen, bei denen nur ein Controller bedient wird, 92 mit den neueren Spielsystemen wie der Wii. Hier muss der Spieler die Bewegung, die im Spiel erwünscht ist, selbst ausführen. Schulter-, Fuß- und Sprunggelenksverletzungen waren bei den interaktiven Spielen signifikant häufiger als bei den klassischen Videospielen. Auch Zuschauer wurden interaktiv öfter in Mitleidenschaft gezogen.

RM =

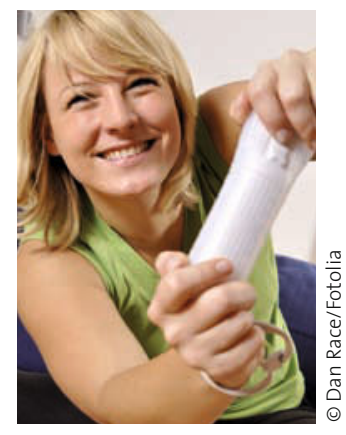

Virtuelle Welten, reales Verletzungsrisiko.

\section{ALKOHOLABUSUS \\ Nicht nur Männer trinken zu viel}

- Bisher war gängige Meinung, dass Alkoholprobleme vor allem Männersache sind. Neue Zahlen, die die Drogenbeauftragte der Bundesregierung, Mechthild Dyckmans, am 5. Oktober vorstellte, belegen, dass dies so nicht stimmt: 370000 der insgesamt 1,3 Millionen Alkoholabhängigen

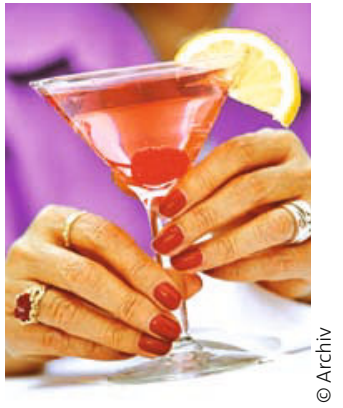

Genuss oder Sucht? sind Mädchen oder Frauen.

Bedenklich ist, dass vor allem junge Frauen exzessiv trinken. So haben bereits vier Fünftel der Mädchen unter 14 Trunkenheitserfahrungen. Die Zahl der 10- bis 15-Jährigen, die mit Alkoholvergiftung in eine Klink eingeliefert wurden, ist zwischen 2000 und 2008 um $170 \%$ auf 2400 gestiegen. Jungen im gleichen Alter waren mit 2100 Fällen vertreten.

Neben den jungen Frauen sind auch ältere Frauen häufiger betroffen: Mit dem Konsum von mehr als 12 Gramm reinem Alkohol am Tag gefährdet jede fünfte Frau zwischen 45 und 54 Jahren ihre Gesundheit. Und Frauen aus akademischen Berufen haben einen stärkeren Hang zum Alkohol als weniger gebildete Frauen. Prävention und Suchthilfe für Frauen sollen künftig einen höheren Stellenwert bekommen, so Dyckmanns.

JM

\section{Röntgenaufnahmen schuld am kindlichen Blutkrebs?}

- Werden Kinder häufiger geröntgt, steigt ihr Risiko, an Leukämie zu erkranken. Vor allem bei der akuten lymphoiden Leukämie (ALL) ist der Zusammenhang zwischen Röntgenuntersuchungen und Erkrankungswahrscheinlichkeit deutlich. Nach Daten der California Childhood Leukemia Study haben Kinder, die an ALL erkranken, etwa zweimal so häufig drei oder mehr Röntgenuntersuchungen hin- ter sich wie gesunde Kinder. Besonders kritisch scheinen Thoraxaufnahmen zu sein. Dagegen wurde kein Zusammenhang gefunden zwischen Röntgenuntersuchungen und der akuten myeloischen Leukämie (AML). Auch eine T-Zell-Leukämie scheint nicht durch häufiges Röntgen begünstigt zu werden.

Es bedürfe weiterer Forschung, um den Zusammenhang zwischen ALL und Rönt- genuntersuchungen zu sichern, meinen die Forscher. Sie raten aber zur Zurückhaltung mit Röntgendiagnostik bei Kindern. Vor allem müsse man sich sehr genau überlegen, ob bei einem Kind ein CT erforderlich sei. Die Strahlenexposition kann dabei 500-mal höher sein als bei einer konventionellen Röntgenaufnahme. RM -

Sarah Yang X-rays linked to increased childhood leukemia risk, Media Relations, 4. Oktober 2010 